\title{
CS07-02
}

\section{WHAT WILL BE THE CONSEQUENCES FROM THE CHANGES IN ICD-11/DSM-V FOR DECISION MAKING IN CLINICAL PSYCHOPHARMACOTHERAPY?}

\section{Bitter}

Department of Psychiatry and Psychotherapy, Semmelweis University, Budapest, Hungary

The preparations of DSM V/ICD-11 are in progress. The frustrations and limitations engendered by the categorical model in psychiatry led to the suggestion of alternative dimensional models of classification, e.g. to the suggestions for a dimensional view of psychosis. However there are strong arguments for keeping the categorical classification maybe with inclusion of new criteria, such as neurocognitive impairment for schizophrenia and even there are suggestions for new categories such as nonaffective remitting acute psychosis.

Therapeutic indications of drugs are developed by the industry and are approved by regulatory agencies (FDA, EMEA). Indications are linked to diagnostic categories as defined by DSM-IV/ICD-11, e.g. one antipsychotic is indicated for the treatment of both schizophrenia and bipolar disorder, while another is only indicated for the treatment of schizophrenia. These indications are used by the insurance industry to manage financing of drug treatment.

Changes in the criteria of a diagnostic category will result in a revision of approval which may include the obligation for new studies. Generic companies are now an important part of the major pharmaceutical markets, however many of them do not have the capacity to launch clinical studies, which may lead to the withdrawal of marketing authorization of important drugs. Clinical studies seem to be unavoidable for licensing recently available drugs for the new diagnostic categories of DSM V/ICD 11. The introduction of diagnostic categories by a dimensional approach may lead to "double book keeping”: a dimensional diagnosis used for the medical practice and a categorical one for the insurance. 